\title{
HYBRID DYSGENESIS IN DROSOPHILA: CORRELATION BETWEEN DYSGENIC TRAITS
}

\author{
P. EGGLESTON and M. J. KEARSEY \\ Department of Genetics, University of Birmingham, P.O. Box 363, Birmingham B/5 2TT
}

Received 23.viii.79

\begin{abstract}
Summary
Crosses between laboratory stocks and extractions from wild populations have recently been shown to produce non-reciprocal genetic aberrations commonly termed hybrid dysgenesis, which appear to arise from a nuclear cytoplasmic interaction. Female sterility is one aspect which has been investigated and both poor egg production (GD sterility) and low hatchability (SF sterility) have been shown to contribute. It has previously been suggested that these characters may have an independent action and causation.

The results presented in this paper however, show a high degree of correlation in the response of SF and GD sterility to various developmental temperature regimes, with both forms of sterility showing an increase as the developmental temperature rises. For each character, the whole of the life cycle appears to be affected by changes in the developmental temperature although two stages were identified as being particularly sensitive. The results therefore suggest that these two characters have a common cause and the relationship between these and other dysgenic traits is discussed.
\end{abstract}

\section{Introduction}

THERE has recently been considerable interest in various forms of " hybrid dysgenesis" in Drosophila melanogaster (Sved, 1976 and Kidwell et al., 1977). This dysgenesis appears as a varying degree of female and male sterility both in terms of egg and sperm production, known as gonadal dysgenesis or GD sterility (Kidwell et al., 1977), and reduced hatchability of eggs, termed SF sterility (Picard et al., 1977). Other dysgenic traits include male recombination, increased mutation rates and a high frequency of meiotic abnormalities (Hiraizumi et al., 1973; Slatko and Hiraizumi, 1973; Cardellino and Mukai, 1975; Yamaguchi, 1976; Woodruff and Thompson, 1977; Thompson and Woodruff, 1978).

It occurs most obviously in the $F_{1}$ progeny of crosses between certain unrelated strains but not in their reciprocals and, as far as they have been studied (Kidwell, 1979) the genetic and cytoplasmic controls of these traits are essentially similar. In general the male parents, referred to as inducers or promotors of dysgenesis, occur in recent extractions from wild populations, while the female parents, referred to as reactive strains, are restricted to certain long-established laboratory stocks (Picard, 1978).

It is clearly of interest to know whether these diverse aberrations have a common or independent cause. To this end Kidwell et al. (1979) examined fecundity, hatchability and male recombination and concluded that there is evidence of independence both in action and causation. This 
conclusion is largely based on the dissimilar patterns shown by these traits when they are studied in different genetic backgrounds and at different temperatures.

In the present paper we wish to show that these apparent discrepancies are largely artefacts produced by the experimental and analytical techniques used. Indeed we attempt to show that the various phenomena correlate very closely.

The paper describes the results of investigating several wild type inbred lines, used as male parents, when crossed to " reactive" testers with respect to the production and hatchability of eggs from the $F_{1}$ daughters. Various temperature regimes have been studied, together with an examination of the developmental age at which temperature responses occur. It should be noted, however, that although egg hatchability has been measured in the conventional way, we have adopted a different approach to measuring egg production from that used in previous GD studies.

\section{MATERIALS AND METHODS}

(i) Strains used

(a) Reactive stocks

y bw st (A)

y bw st (B)

These are two long inbred stocks carrying the following recessive markers on the three major chromosomes.

$$
\begin{array}{rlrl}
y & =\text { yellow (body) } & \mathrm{X}-0.0 \\
b w & =\text { brown (eye) } & 2-104.5 \\
s t & =\text { scarlet (eye) } & & 3-44.0
\end{array}
$$

$y$ bw st (A) is known to be a strongly reactive stock while on previous evidence $y$ bw st (B) was thought to be neutral or only slightly reactive (Kearsey et al., 1977).

\section{(b) Potential inducer lines}

These include 16 inbred lines derived from the Texas population. This population was established in 1965 from flies captured at Austin, Texas and the inbred lines have been established by sib-mating for more than 200 generations prior to their use in these experiments (Linney et al., 1971). Also included was Samarkand, a long-established, inbred, wild type laboratory stock.

(ii) Designation of crosses

Following the designation of Kidwell et al. (1979) crosses are referred to as follows:

Cross A

y bw st (우) $\mathrm{X}$ wild-type (숭)
Cross B

wild type (우) $\mathrm{X}$ y bw st $\left(\hat{0} \delta^{\hat{\sigma}}\right)$

Only Cross A is expected to produce dysgenic progeny and it can therefore be described as a "reactive " cross. Cross B can be referred to as the "control " cross. 
(iii) Methods of assessing female sterility

For the experiments described in this paper, flies were allowed to develop at several temperatures. However, after eclosion all scoring was performed at a temperature of $25^{\circ} \mathrm{C}$.

\section{(a) SF sterility-measurement of hatchability}

After eclosion, replicate samples of $F_{1}$ females were transferred to fresh culture media and allowed to mate with their brothers for three days at $25^{\circ} \mathrm{C}$, in order to ensure insemination. A standard male is not required since the choice of male parent has been shown to be unimportant (Bucheton et al., 1976). Experience has shown that the most reliable estimates of hatchability are obtained if eggs are sampled on the basis of one egg per female; therefore attempts were made to base each measurement on at least 25 females, from which a sample of 25 eggs would be taken. After mating for three days the flies were transferred to fresh tubes containing plastic partitions coated with blackened starch paste and the females were allowed to lay overnight for 15-16 hours. Twenty-five eggs were then transferred to blackened 2 per cent agar in a petri dish. Hatchability was scored after 30 hours and the process was repeated with the same females in order to obtain replicate observations.

\section{(b) GD sterility-measurement of egg production}

Females were assessed for egg production between the two measurements of hatchability described above. The fact that the females have been laying overnight before egg production is scored, avoids any bias due to the accumulation of eggs in utero. After the number of females in each replicate sample had been scored, the flies were placed for one hour at $25^{\circ} \mathrm{C}$ in fresh tubes containing the blackened partitions. The flies were then removed, the number of eggs on the partition counted and the process repeated. Egg production is recorded in terms of the number of eggs per female per hour.

\section{(iv) Transformation of the data}

Whenever it was desired to carry out analyses of the data which depended upon tests of significance, the raw data had to be transformed to improve the normality of the distribution. To this end hatchability percentages were converted to angles, with values of 0 and 100 per cent being increased and decreased respectively by $\frac{1}{4 N}$ (following Bartlett, 1947). In every case $N$ was 25. Examination of the egg production data showed that there was a high correlation between the mean and the variance $(\hat{r} \simeq 0.9)$. The appropriate transformation in this event is a conversion to square roots and consequently this was applied to each GD observation separately.

\section{(v) Experimental design}

In each of the experiments described in this paper replicate cultures of the required crosses were set up. For each inducer in the inbred line survey, two replicate sets of parents were introduced into bottles for both Cross A 
and Cross B. For the temperature shift experiments, eggs were collected from the parents and raised in tubes at low fixed densities, in order to achieve synchrony of development. Temperature treatments were based on sets of 10 replicate cultures. All experiments were individually randomisedreplicate cultures being scored separately and on more than one occasion.

\section{(vi) Culture medium}

All crosses were raised on standard oatmeal-molasses-agar medium in either $\frac{1}{3}$-pint milk bottles or $2.5 \mathrm{~cm} \times 7.5 \mathrm{~cm}$ vials.

\section{The efFects of DeVelopmental temperature on SF and GD STERILity}

A survey of the wild type inbred lines available in this department was carried out in order to assess their ability to induce SF and GD sterility when crossed to $y$ bw st (A), a known reactive stock. The inbred lines used included 16 extracted from the Texas population and one extracted from the Samarkand population. Of these lines, Samarkand and Tex I were also crossed to $y$ bw st (B). The purpose of these additions to the survey was to see if different populations and stocks behaved in a similar fashion. For each inbred line Cross A and Cross B were raised at each of four developmental temperatures, $20^{\circ}, 23^{\circ}, 25^{\circ}$ and $28^{\circ} \mathrm{C}$, and the female progeny were assessed for SF and GD sterility as detailed in materials and methods. Several interesting results were revealed by the survey.

Firstly, every inbred line tested proved capable of inducing both SF and GD sterility when crossed to $y$ bw st (A). This is consistent with previous reports (Bucheton et al., 1976) that all lines recently extracted from the wild are capable of acting as inducers. The second potentially reactive stock, $y$ bw st (B), also produced SF and GD sterility when crossed as the female parent to Samarkand and Tex I, although at a lower level than crosses involving $y$ bw st (A). It can therefore be considered as a weaker reactive stock and this is consistent with previous reports of variation in reactivity levels (Bucheton et al., 1976). The Samarkand inbred line behaved in much the same way as did the Texas lines and this consistency of populations was confirmed by a less detailed and unpublished study of inbred lines in this laboratory involving five other populations, Oregon, Florida, $6 \mathrm{C} / \mathrm{L}$, Wellington and Edinburgh.

Secondly, a general pattern of egg production and hatchability arose from the survey. Cross A generally shows a sigmoidal decline in hatchability with increasing developmental temperature, whereas the hatchability of eggs produced by Cross B progeny remains relatively constant at $90-100$ per cent. The mean control hatchability over the whole experiment (fig. 1), was approximately 95 per cent.

Measurement of egg production proved more difficult. Due to the size of the survey it was necessary for each developmental temperature to be

FIG. 1.-The effect of developmental temperature on GD sterility (solid line) and SF sterility (broken line) for each of three Texas inducing lines, $(a),(b)$ and $(c)$ representing Tex 6 , Tex 19 and Tex 9 respectively. In each case A represents the reactive cross (Cross A) and $\mathrm{B}$ the control cross (Cross B).

The GD (egg production) scale shows the percentage reduction of cross A from Cross B while the SF scale depicts percentage hatchability. See text for details. 

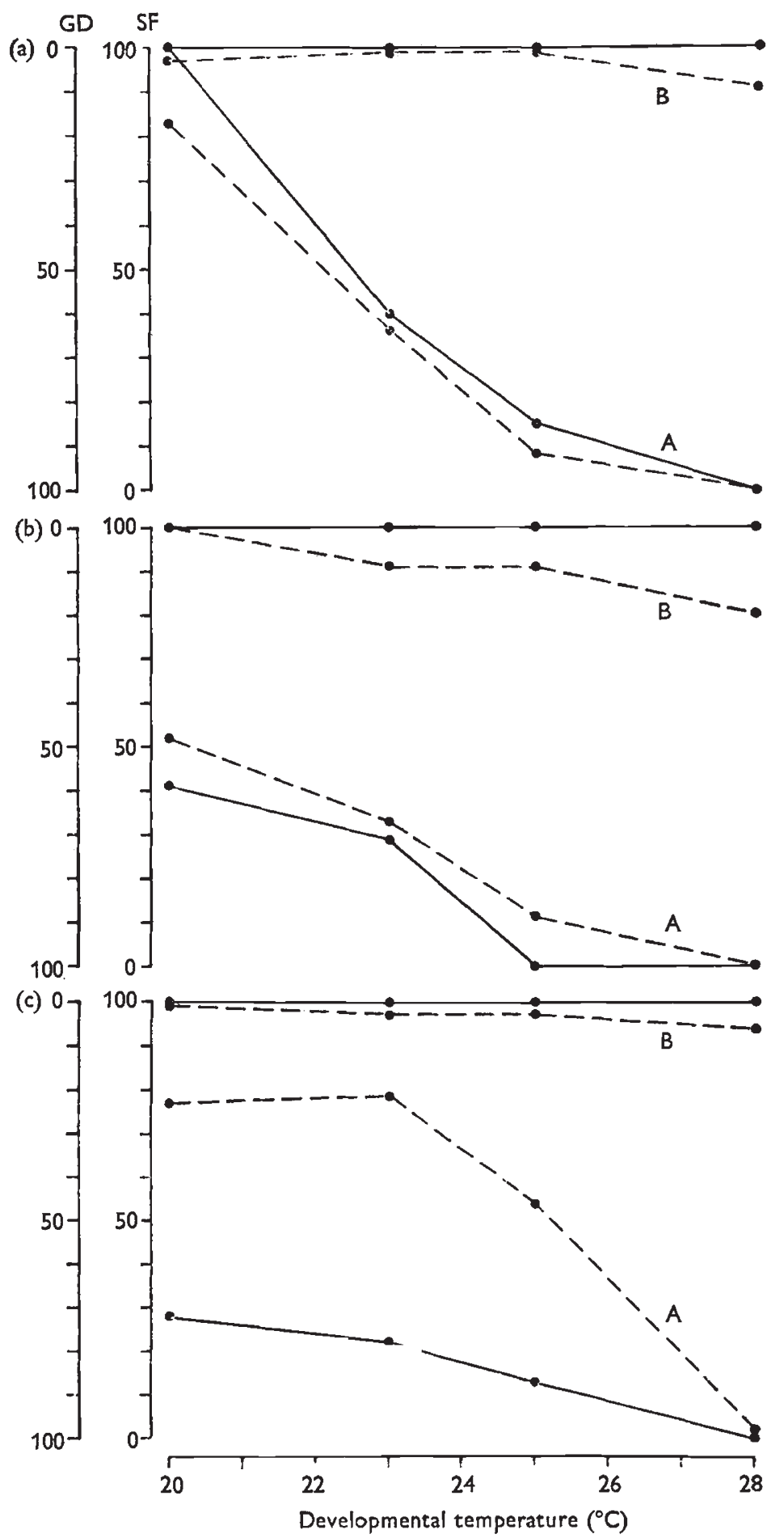

Fig. 1.-Caption on facing page. 
studied on a different occasion and this led to problems when comparing GD sterility levels between experiments. This character is very sensitive to environmental fluctuations, so much so that it was found necessary to draw conclusions from within rather than between experiments. Experience has shown that providing Gross A and Gross B (for any particular inducing line) are raised and scored under the same conditions, a useful measure of the GD sterility level can be obtained by tabulating the Cross A "reactive" score as a percentage reduction from the Gross B "control" score.

The survey data, when treated in this way, show that egg production of Cross A daughters also shows a sigmoidal decline with increasing developmental temperature (fig. 1). What is more, the decline is very similar in many respects to that of egg hatchability. Several pieces of evidence now indicate that SF and GD scores correlate extremely well in their response to developmental temperature. Over and above the general pattern of sterility, there was marked variation in the way egg production and hatchability declined, depending on the particular inducing line involved. There appear to be at least three major types of pattern and a larger survey may well show a continuum between these extremes. The results of crosses which

TABLE 1

Two way analysis of variance of the differences between the control and the reactive scores. GD observations are square roots of the number of eggs per female per hour and SF observations are in angles. All entries are based on individual observations and the theoretical error is calculated as $820 \cdot 7 / \mathcal{N}$, where $\mathcal{N}$ is 25 .

\begin{tabular}{|c|c|c|c|c|c|c|c|c|}
\hline \multirow[b]{2}{*}{ Item } & \multicolumn{4}{|c|}{ SF } & \multicolumn{4}{|c|}{ GD } \\
\hline & $\mathrm{DF}$ & MS & VR & $\mathbf{P}$ & DF & MS & VR & $\mathrm{P}$ \\
\hline Temperature $(\mathrm{T})$ & 3 & $10267 \cdot 69$ & $260 \cdot 77$ & $<1 \%$ & 3 & $5 \cdot 44$ & $16 \cdot 15$ & $<1 \%$ \\
\hline Lines (L) & 16 & $718 \cdot 74$ & $3 \cdot 88$ & $<1 \%$ & 15 & $1 \cdot 98$ & $3 \cdot 00$ & $<1 \%$ \\
\hline $\mathrm{L} \times \mathrm{T}$ & 48 & $185 \cdot 06$ & $4 \cdot 70$ & $<1 \%$ & 45 & 0.66 & 1.96 & $<1 \%$ \\
\hline Error & 432 & $39 \cdot 38$ & $1 \cdot 20$ & $5-1 \%$ & 396 & $0 \cdot 34$ & & \\
\hline Th. error & $\infty$ & 32.83 & & & & & & \\
\hline
\end{tabular}

display these three patterns are shown in fig. 1. The first type is best illustrated by Tex 6 (fig. 1 $(a)$ ). Both egg production and egg hatchability from Cross A approach the control values as the developmental temperature decreases, and they do so in very close agreement with one another. Tex 19 (fig. $1(b)$ ) displays the second pattern where both egg production and egg hatchability from Cross A remain relatively low over the whole developmental temperature range. There is still however, a sigmoidal decline of both characters as the developmental temperature increases, and once again there is a marked similarity in the response of the two types of sterility. The third pattern is displayed by Tex 9 (fig. 1(c)), where although egg hatchability from Cross $\mathrm{A}$ approaches the control value at lower, more permissive, developmental temperatures, egg production remains relatively low when compared to the control cross. The response of the two characters to developmental temperature is again correlated. There were no examples of inducing lines where the hatchability from Cross A remained low while egg production increased with decreasing developmental temperature.

It is evident from this pictorial representation of the survey that the response of SF and GD sterility to developmental temperature is very similar, 
regardless of the specific pattern involved. An analysis of variance of the survey data (table 1), based on the differences between the Cross B and the Cross A scores, confirmed that not only were there highly significant differences between lines and between temperatures, but that the various lines also responded differently to temperatures. That is, the highly significant lines by temperatures interaction term for both SF and GD provides evidence for the existence of several patterns of response to developmental temperature. For any particular pattern of response there is a high correlation between the SF and the GD scores as they decline with increasing developmental temperature.

In order to confirm this similarity of response, correlation coefficients and regressions of GD onto SF were calculated for each temperature (table 2 ). In every case there is a highly significant regression of GD on SF, and the mean correlation coefficient over the four temperatures is 0.73 . Thus, on average, $0.73^{2}$ ( 53 per cent) of the variation among GD scores can be

TABLE 2

Regressions of GD onto SF scores for each of the four developmental temperatures. All entries are based on individual observations of egg production (transformed to square roots) and hatching percentages (transformed to angles). The error term is that obtained from pooling the error among individual observations from each of the four temperatures. Correlation coefficients are given for each temperature, the mean coefficient being 0.73 .

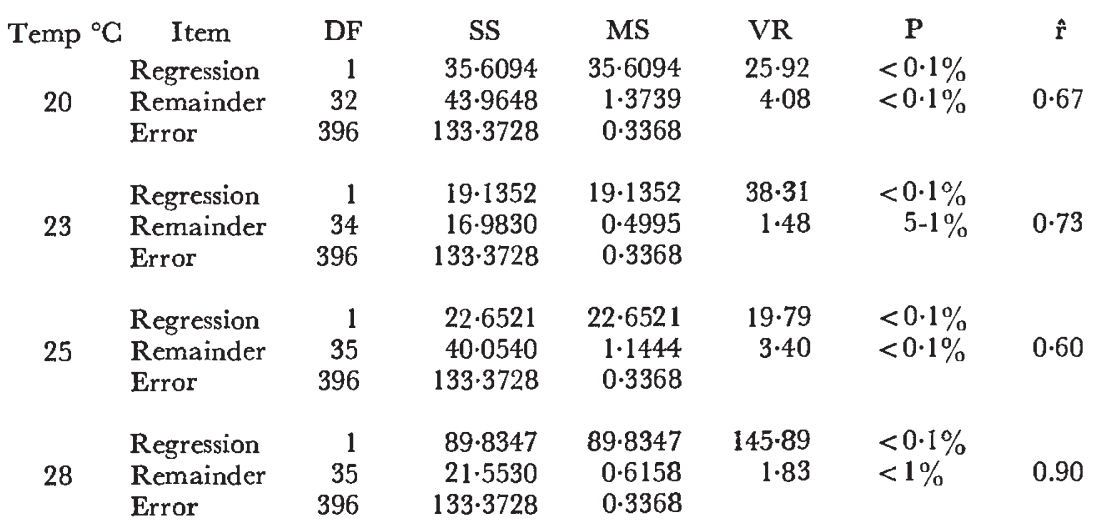

accounted for by the SF variation. However, the GD data cannot be explained fully in terms of the SF data, since the remainder mean squares from each of the four regressions are significantly greater than the error among individual GD observations, which is the appropriate error in this case.

Thirdly, the survey showed that "reactive" crosses never achieve the levels of egg production and hatchability set by the " control " crosses, over the range of developmental temperatures used. This has been shown consistently for all inducing lines and reactive stocks surveyed. In general, the progeny from Cross A do better at lower temperatures $\left(18-20^{\circ} \mathrm{C}\right.$ ) but the highest levels of egg production and hatchability they achieve depend upon the inducer used. Evidently, the underlying cause of the sterility cannot be completely overridden regardless of the temperature at which the flies develop. 


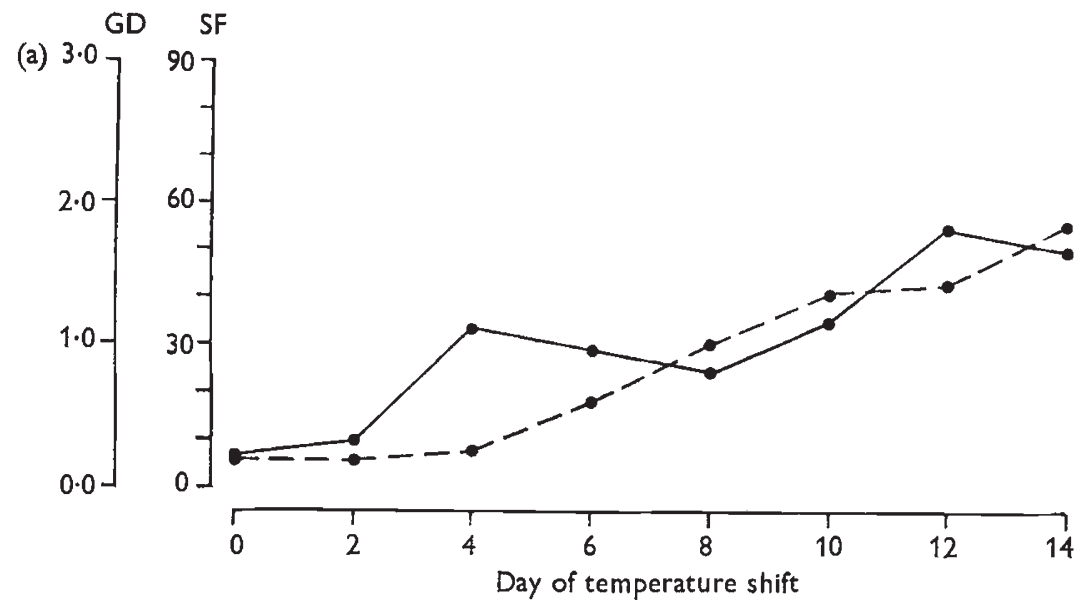

(b) 3.0
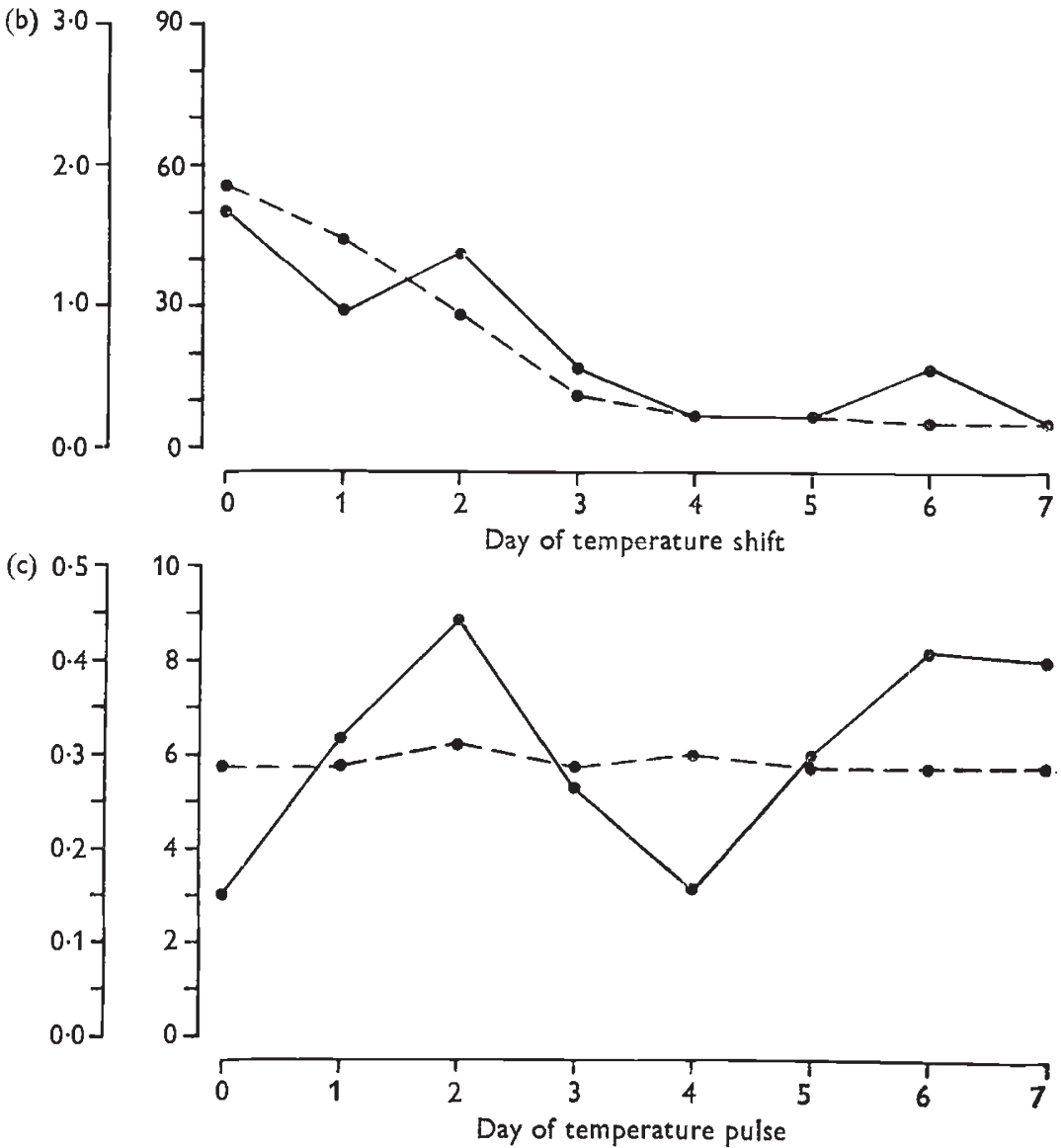

Fig. 2.- Results from the temperature shift experiments involving transfers from $20^{\circ}$ to $28^{\circ} \mathrm{C}(a)$ and from $28^{\circ}$ to $20^{\circ} \mathrm{C}(b)$. The temperature pulse experiment is summarised in $(c)$. In each case GD sterility (solid line) is presented as the square root of the egg production per female per hour and SF sterility is presented in angles. See text for details. 


\section{Examination of THE Temperature SENSitive STAges}

Individually randomised cultures of $y$ bw st $(\mathrm{A}) \times$ Tex I were subjected to reciprocal temperature shifts between the permissive $\left(20^{\circ} \mathrm{G}\right)$ and the restrictive $\left(28^{\circ} \mathrm{C}\right)$ developmental temperatures. Cultures raised at $28^{\circ} \mathrm{C}$ display both SF and GD sterility, those raised at $20^{\circ} \mathrm{C}$ display little of either form of sterility. The experiment was designed to find out when developing flies were sensitive to these changes in developmental temperature and also whether (as suggested in the previous section) SF and GD sterility were induced by the same developmental conditions and at the same time. Approximately 20,000 eggs were collected overnight from the parents, the females laying for 15-16 hours. These eggs were transferred at a density of 100 per tube to 160 tubes at random. Half of these tubes were placed at $20^{\circ} \mathrm{C}$, the other half at $28^{\circ} \mathrm{C}$, and 10 replicate tubes chosen at random were transferred every day from $28^{\circ}$ to $20^{\circ} \mathrm{C}$ where they were allowed to complete their development. From the other group, 10 randomly chosen tubes were transferred every 2 days from $20^{\circ}$ to $28^{\circ} \mathrm{C}$, the flies again being allowed to complete their development at the new temperature. As the sets of cultures emerged, they were assessed for SF and GD sterility as before. From the results summarised in fig. 2(a), (b) it can be seen that the SF and GD scores follow one another very closely, and in fact the correlation between them is

TABLE 3

Regressions of GD onto SF scores from the reciprocal temperature shift experiment. The design consisted of 16 sets of 10 replicate cultures each scored on two occasions, which accounts for the degrees of freedom of the error term. The GD observations are square roots of the number of eggs per female per hour and the SF observations are angles. All entries are based on individual observations.

$\begin{array}{lrrrrr}\quad \text { Item } & \text { DF } & \text { SS } & \text { MS } & \text { VR } & \text { P } \\ \text { Regression } & 1 & 68 \cdot 7493 & 68 \cdot 7493 & 36 \cdot 45 & <1.0 \% \\ \text { Remainder } & 14 & 26.4026 & 1.8859 & 10 \cdot 34 & <0 \cdot 1 \% \\ \text { Error } & 288 & 52.5312 & 0.1824 & & \end{array}$

0.73 which is exactly the same value as that obtained from the survey. The regression of GD on SF is highly significant (table 3) but the remainder mean square is also significantly greater than the appropriate error term. This reflects the same problem that occurred in the inbred line survey, in that although there is a great deal of similarity between the responses of $\mathrm{SF}$ and GD, and a high correlation, there is still a proportion of the variation among GD scores which can not be explained in terms of the SF variation. Some possible explanations for this are given in the discussion.

The second major point to note is that there does not appear to be a particular stage in the life cycle which is sensitive to the temperature shifts. Fig. 2(a), (b) show that developing flies are sensitive to being placed at the restrictive temperature $\left(28^{\circ} \mathrm{G}\right)$ throughout their life cycle. It can also be seen that flies which spend any part of their life cycle at $28^{\circ} \mathrm{C}$, do worse than flies which complete their whole development at the permissive temperature $\left(20^{\circ} \mathrm{C}\right)$. The shift from $28-20^{\circ} \mathrm{C}$ (fig. $2($ b $)$ ), typically produces a sharper image of the response than $20-28^{\circ} \mathrm{C}$ (fig. $2(a)$ ) because the shorter life cycle produces less variation in developmental age at the time of scoring. It can 
be seen from fig. $2(b)$ that there is a rapid increase in sterility in the larval stages, particularly the earlier ones. This is therefore a slightly more sensitive stage, and time spent at $28^{\circ} \mathrm{C}$ here will have a more dramatic effect than elsewhere. This is confirmed by fig. 2(a) where the greater response of this stage is evident from the flattening at the start of the curve which indicates a depression of egg production and hatchability. That is, the shift to $28^{\circ} \mathrm{C}$ produces a higher level of sterility if it occurs when the developing flies are first or second instar larvae than if it occurs at another stage. The results also suggest that there is a period during the pupal phase which is particularly sensitive to temperature shifts. The evidence for this comes mainly from the rise in egg production between days 5 and 6 of fig. $2(b)$. This effect has now been shown in three separate experiments. It is not known why the SF scores do not respond in the same way, but flies developing under these conditions produce very few eggs and the failure to detect a rise in hatchability could well be due to poor sampling. The analogous developmental stage for fig. 2(a) (10-12 days) shows a flattening of the SF curve which could indicate a stage particularly sensitive to the shift to $28^{\circ} \mathrm{C}$ but the egg production rises rapidly instead of following the SF scores. This, together with the failure of the SF scores to respond between days 5 and 6 (fig. 2(b)) suggests that a large part of the increase in egg production during this stage could be an artefact of the temperature shock, and not an integral part of GD sterility. This is currently being studied in an attempt to resolve the question.

In order to gain more information concerning the response to temperature shifts, a second experimental design was developed. This involved raising eighty individually randomised cultures of the cross $y$ bw st $(\mathrm{A}) \times$ Tex $\mathrm{I}$ at $28^{\circ} \mathrm{C}$. After successively longer periods at $28^{\circ} \mathrm{C}$ ten randomly chosen replicates were placed at $20^{\circ} \mathrm{C}$ for twenty-four hours and afterwards returned to $28^{\circ} \mathrm{C}$. The experimental technique was similar to that of the previous experiment in that eggs were collected overnight and then transferred to tubes, this time at a density of 200 per tube in an attempt to increase the number of emerging females. Upon emergence, the $F_{1}$ females were assessed for SF and GD sterility as before and the results are summarised in fig. $2(c)$.

It can be seen that the results are in close agreement with the predictions from the previous experiment. The general picture is one of high sterility, since the progeny have spent almost seven days at the restrictive temperature $\left(28^{\circ} \mathrm{C}\right)$. However, the two sensitive stages suggested by the previous experiment are also shown up here by increases in fertility. There is an obvious rise in both egg production and hatchability during the earlier larval stages, even though the latter falls short of statistical significance. Once again there is a significant rise in egg production occurring during the pupal phase, at exactly the same developmental stage as in the previous experiments, but hatchability does not respond in the same way. This is thought to provide further evidence that increased egg production during this phase is a consequence of the temperature shock and not an integral part of GD sterility. Further arguments for this are advanced in the discussion. It should be noted that this experiment provides confirmation that the whole of the life cycle is sensitive to temperature since the mean egg production of those sets which received a $20^{\circ} \mathrm{C}$ pulse is in every case higher than the control set (day 0 ), which developed entirely at $28^{\circ} \mathrm{C}$. 


\section{Discussion}

The experiments described in this paper show a definite pattern of response of SF and GD sterility to developmental temperature and provide evidence that the two characters have a common causation, essentially as a result of the method of measuring GD sterility. In the earlier studies of GD sterility (Kidwell et al., 1979) the fertility of a particular genotype was scored simply as the proportion of females which laid eggs, no account being taken of whether they laid one or 20. Such a procedure imposes an arbitrary discontinuity onto a continuously varying trait and, in so doing, automatically conceals the large variance in egg production among flies classed simply as " egg laying". Consider the case of egg production per female rising sigmoidally over a range of treatments, (such as decreasing developmental temperature), as we find in the present data. If we were to measure the proportion of females which laid one or more eggs we would find a rapid rise in fertility over a very short span of developmental temperature. This is in fact the case reported by Kidwell et al. (1979). The two patterns of GD response would thus appear quite different except at the start and end of the curves, and this would suggest quite different types of control even though they are measures of the same variable. We believe that this scalar effect entirely accounts for the discrepancies between Kidwell's results and our own.

In her report on GD sterility Kidwell concluded that although there were marked similarities between the I-R system (SF sterility) of Picard et al. (1977) and the P-M system (GD sterility) of Kidwell et al. (1977) the two forms of sterility were independant in action and causation. This conclusion was based on two main pieces of evidence.

(a) GD sterility rises over a very narrow critical range of temperatures, whereas the response of SF sterility is more gradual.

(b) There is a narrow range of developmental age during which GD sterility responds to developmental temperature whereas SF sterility responds over a broad age range.

Both of these differences, however, can be explained by the artificial foreshortening of the response curve explained above. There is in fact a progressive increase in both forms of sterility with increasing developmental temperature, and all stages of the life cycle are sensitive to developmental temperature changes, although two stages have been identified as being particularly sensitive. The scalar effect also explains why Kidwell observed different response patterns between GD sterility and two other traits, male recombination and transmission ratio distortion. The latter two show a response over a very broad developmental temperature range whereas GD sterility was observed to have a narrow critical range. Had egg production been scored as a continuous variable, the three responses would probably have been very similar.

It can be argued that the higher critical temperature required for male sterility also stems from the technique employed. Thus, for a female to be declared sterile, it has to lay no eggs, while for a male to be declared sterile, all four of its mates must fail to lay eggs. It follows that since the test for male sterility is more exacting it will only appear under more stringent conditions, that is at higher developmental temperatures. The results

$$
44 / 2-\mathrm{G}
$$


reported in this paper suggest that there could well be a common response to developmental temperature for all of these dysgenic traits.

The results show that although GD and SF sterility respond to developmental temperature in a very similar way, there is still a proportion of the variation among GD scores that cannot be accounted for by the SF variation. The majority of this is due to the rise in egg production associated with a temperature change during the pupal phase (fig. 2), which is not accompanied by a rise in hatchability. Differences between GD and SF scores in other parts of the life cycle can be attributed to the large standard errors of GD measurements, since previous experiments have produced GD values both above and below the $\mathrm{SF}$ scores. The rise in egg production during the pupal phase has, however, been shown consistently in all experiments and is never accompanied by a rise in hatchability. A review of the relevant literature shows that this stage is the time of meiosis in the developing pupa with both recombination and DNA replication taking place. It has been shown that exposure to high temperature at this stage causes an increase in both recombination (particularly centromeric) and DNA replication (Grell, 1972) and this may well be a cause of increased egg production. There is no published evidence for a similar rise in egg production for flies raised at a high temperature and given a low temperature shock, but this may well prove to be the case. The results of our experiments suggest that a temperature shock during this phase causes an increase in egg production which is not an integral part of GD sterility, but a consequence of the temperature shock. Experiments are now being conducted with nondysgenic females to see if temperature shock during this phase causes increased egg production while leaving hatchability unaffected. If this is the case then the actual GD score will move nearer the SF score, improving the overall correlation. The exact relationship between this rise in egg production and the events of meiosis is not clear, but the onset and duration of the two phenomena are extremely similar and this suggests that the link is not merely coincidental.

From the results presented in this paper, we therefore conclude that SF and GD sterility have a common cause and a very similar response to developmental temperature. Moreover, from the information available on the other dysgenic traits, it seems likely that far from being diverse, independent phenomena, they are all in fact aspects of the same nuclearcytoplasmic interaction, with a common causation. If this is true then future research into hybrid dysgenesis may well be able to concentrate on those traits which can be measured easily and accurately, such as egg hatchability.

Acknowledgments.-We are grateful to Dr P. D. Caligari for his helpful discussion and to the technical staff of the department for their assistance. We acknowledge the financial assistance of the Science Research Council. All the computations in this paper were carried out on Birmingham University's ICL 1906A computer.

\section{REFERENCES}

BARTLETT, M. s. 1947. The use of transformations. Biometrics, 3, 39.

BUCHETON, A., LAVIGE, J. M., PICARD, G., AND L'Heritier, PH. 1976. Non-mendelian female sterility in Drosophila melanogaster: quantitative variation in the efficiency of inducer and reactive strains. Heredity, 36, 305-314.

CARDELlino, R. A., AND MUKAI, T. 1975. Mutator factors and genetic variance components of viability in Drosophila melanogaster. Genetics, 80, 567-583. 
GRell, R. F. 1972. Recombination and DNA replication in the Drosophila melanogaster oocyte. Genetics, 73, 87-108.

hiRAizumI, y., SLATKo, B., LANGley, C., AND NIll, A. 1973. Recombination in the Drosophila melanogaster male. Genetics, 73, 439-444.

Kearsey, M. J., Williams, W. R., Allen, P., AND COUlter, F. 1977. Polymorphism for chromosomes capable of inducing female sterility in Drosophila. Heredity, 38 (1), 109115.

KIDWELL, M. G. 1979. Hybrid dysgenesis in Drosophila melanogaster: the relationship between the P-M and I-R interaction systems. Genetical Research, 33, 205-217.

KIDWELL, M. G., AND NOVY, J. B. 1979. Hybrid dysgenesis in Drosophila melanogaster: sterility resulting from gonadal dysgenesis in the P-M system. Genetics, 92, 1127-1140.

KIDWELl, M. G., KIDWELl, J. F., AND sved, J. A. 1977. Hybrid dysgenesis in Drosophila melanogaster: a syndrome of aberrant traits including mutation, sterility and male recombination. Genetics, 86, 813-833.

LINNEY, R., BARNES, B. W., AND KEARSEY, M. J. 1971. Variation for metrical characters in Drosophila populations. Heredity 27 (2), 163-174.

PICARD, G. 1978. Non-mendelian female sterility in Drosophila melanogaster: sterility in the daughter progeny of SF and RSF females. Biologie Cellulaire, 31, 235-244.

PiCARD, G., LAVIGE, J. M., BUChETON, A., AND BREgLiano, J. c. 1977. Non-mendelian female sterility in Drosophila melanogaster: physiological pattern of embryo lethality. Biologie Cellulaire, 29, 89-98.

SLATKo, B. E., AND HIRAIzUMI, y. 1973. Mutation induction in the male recombination strains of Drosophila melanogaster. Genetics, 75, 643-649.

sved, J. A. 1976. Hybrid dysgenesis in Drosophila melanogaster: a possible explanation in terms of spatial organisation of chromosomes. Aust.7. Biol. Sci., 89, 375-388.

WOODRUPF, R. C., THOMPSON, J. N. JR. 1977. An analysis of spontaneous recombination in Drosophila melanogaster males. Heredity, 38 (3), 291-307.

YAMAGUCHI, o. 1976. Spontaneous chromosome mutation and screening of mutator factors in Drosophila melanogaster. Mutation Research, 34, 389-406.

THOMPSON, J. N. JR., AND WOODRUFF, R. C. 1978. Chromosome breakage: a possible mechanism for diverse genetic events in outbred populations. Heredity, 40, 153-157. 